\title{
Improvement and Optimization of Workshop Workflow
}

\author{
Huimin Duan \\ School of Management, Shanghai University, Shanghai 200444, China. \\ duanhuimin1994@126.com
}

Keywords: Optimization, workshop workflow, significantly improved.

\begin{abstract}
This paper uses the causal analysis method to analyze the causes of the low efficiency of the three-phase four-wire meter, and uses the fish bone map to visually display the various influencing factors and find out the key factors. Through analysis and improvement, the assembly time has been shortened, then the production efficiency has been significantly improved, and the goal of maximizing the production efficiency of the company has been achieved.
\end{abstract}

\section{Introduction}

Production system optimization is an important part of production management. As an important part of production management, enterprise production system evaluation develops with the development of production management content. The continuous, stable, and healthy development of an enterprise will inevitably require that it closely follows the changes in the market environment and changes the internal production management and operation mechanisms of enterprises to meet the demands of the new situation on the internal organization, operating structure, and management system of the enterprise. This will enable the company to gain new competitive advantages and make great progress on a brand new strategic platform, which will in turn enable the entire company to develop into a new strategic platform.

Yantai Dongfang Wisdom Electric Co. Ltd. specializes in R\&D, production and sales of electric energy measurement and power information acquisition and management systems. It is a national high-tech enterprise, a software company in Shandong Province and Yantai City Engineering Technology Center.

DTZY178-Z series three-phase smart energy meter integrates metering, display, communication, monitoring, and cost control functions. It can be widely used in electric gateways, power plants, transformer stations, enterprises and institutions for comprehensive energy measurement and management and industrial User multi-rate energy metering.

The design idea of this design is to clarify the main problems existing in the field through actual site visits, and then use the theoretical methods of industrial engineering to conduct comprehensive analysis and implement site improvements.

\section{Status Survey and Improvement}

\subsection{Problems with Site Management.}

There are two main problems in the company's on-site management: First, the implementation of $5 \mathrm{~S}$ is not enough, the on-spot items are disorderly and the waste is serious. Tools, materials, finished products, semi-finished products, and sundries are stacked in a random manner, various necessities and non-essential items are randomly placed on the operating table, and the work table is stained more. The on-site investigation also found that the company's waste was serious, such as waste of defective products due to waiting, handling, and improper operation, and the irrational production processes and poor coordination of production could be the main causes. Besides, there are waste of inventory and manufacturing waste caused by inefficient production organization and a large product backlog. The second problem is the irrational production process.

Through on-site observation, it was found that during the production and assembly process of three-phase four-wire smart meters, there were many problems like slack, unreasonable assembly 
process and the assembly time was too long. In addition, in the production process, material shortages, unreasonable

equipment design and too much unnecessary handling also reduce the efficiency.

\subsection{Current Situation Causal Analysis - Fishbone Analysis.}

The fishbone diagram, which is similar to the tree diagram, is always used to analyze the situation, clear ideas and find the problems.To solve the problem, the fishbone diagram is a tool to help the system fully understand the problem and refine the problem. It is an analysis method through the phenomenon to see the essence. For the problems in the on-site production process, according to the correlation between them, we can establish the fishbone diagram in Figure 1 below:

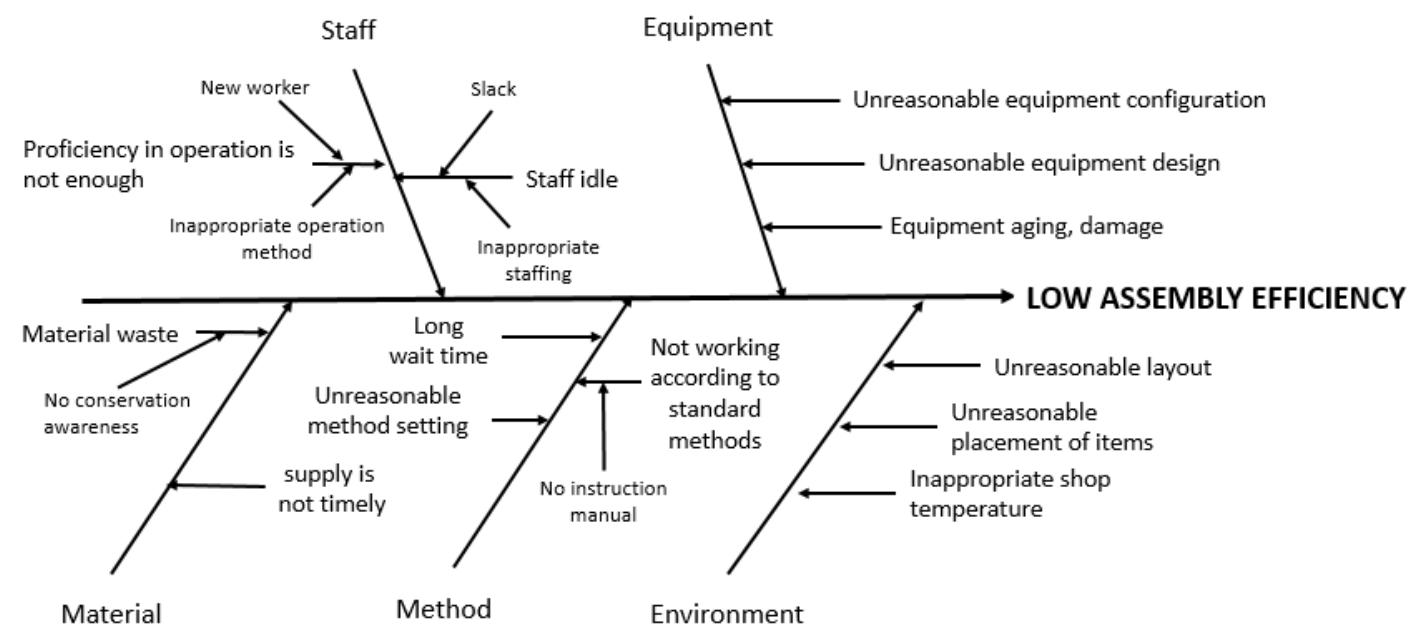

Figure 1. The causal analysis diagram of the assembly of three-phase four-wire smart electronic watch

\section{Production Improvement}

According to the causal analysis (fishbone diagram analysis), the typical seven major waste (as shown in the following table, Table 1) and the auxiliary plan for improvement, an improvement plan was proposed.

Table 1. The typical seven major waste

\begin{tabular}{ccc}
\hline Type & Reason 1 & Reason 2 \\
\hline Overproduction & Production according to plan & Fixed mass production \\
Waiting & Improper arrangement of work & Machine maintenance, \\
Carrying & Unreasonable layout of & production site \\
& Unreasonable processing & morproduction, placement, accumulation, \\
procedures & Defective rework \\
Action & Unreasonable action design & Excessive operation \\
Inventory and & Inaccurate market demand & Overdue procurement, production \\
warehousing & information & Poor technical level, poor quality \\
Product quality & Poor inspection, non-standard & operation
\end{tabular}

Based on on-site observations, the seven major wastes, and the auxiliary plan, the following improvements have been made:

Rearrange the assembly line of the DTZY178-Z three-phase four-wire smart meter assembly to improve facility planning, eliminate excess handling, and reduce long-term handling.

By arranging the power board commissioning point after the fixed three-phase line, it is possible to reduce unnecessary long-term handling.

Change the packing process from one person supplying one assembly line into one person supplying two assembly lines. 
Fixing the cable and CPU board at the same time can achieve the balance of the production line. And remember do not separate the two steps of fastening the shell and the shell can also keep the balance of the production line and avoid excessive waiting.

\section{Conclusion}

After the above analysis and improvement, it was concluded that the improved process included a total of 40 processes, which resulted in the reduction of 3 unnecessary processes compared with before; the improved handling was reduced from the original 9 to the current 5; the improved inspection process consisted of The original 7 steps are reduced to the current 6 steps. The reduction of the processing and assembly process and the merger of the three processes took a total of 1993.97 seconds to reduce the time; the five processes reduced by the transport from the original 252.39 seconds to the current 53.67 seconds, a total reduction of time 198.72 seconds, the distance from the original 180.4 meters Up to the current 40.6 meters, the total transport distance was reduced to 139.8 meters; the reduction in the number of inspection steps increased by 190.02 seconds. Through the improvement, a total time saving of 2002.67 seconds was used, a total reduction in the handling distance was 139.8 meters, and the savings were significant. Through the improvement, the time is saved, the handling distance is greatly shortened, the production efficiency is increased, and the intended purpose is achieved.

Through the investigation of the actual management situation of Yantai Dongfang Wisdom Electric Co. Ltd., using the theoretical methods of industrial engineering, we have obtained time and space, and reached the goal of high efficiency, high quality, low consumption, and safety. This has improved the product quality of the company, shaped the company's good image, and improved the overall efficiency of the company's production.

\section{Acknowledgments}

I am grateful for many detailed and helpful comments from my tutor. And I thank the company for providing me the opportunity to investigate in the workshop.

\section{References}

[1]. Tong K N, Xu K L, Zheng Y Q. Sequencing mixed-model flexible assembly lines with variable launching intervals [J]. Journal of Shanghai Jiao tong University (Science), 2013, 18(4):460-467.

[2]. Ding J Y, Song S, Wu C. Carbon-efficient scheduling of flow shops by multi-objective optimization [J]. European Journal of Operational Research, 2015, 248(3).

[3]. Kasich M. Multi-objective genetic algorithm for energy-efficient job shop scheduling [J]. International Journal of Production Research, 2015, 53(23):7071-7089.

[4]. Claudia E, Maria J. Energy efficiency, robustness, and make span optimality in job-shop scheduling problems [J]. Ai Edam Artificial Intelligence for Engineering Design Analysis \& Manufacturing, 2015, 30(3):300-312.

[5]. Zhang R, Chong R. Solving the energy-efficient job shop scheduling problem: a multi-objective genetic algorithm with enhanced local search for minimizing the total weighted tardiness and total energy consumption [J]. Journal of Cleaner Production, 2016, 112:3361-3375.

[6]. Li C, Xiao Q, Tang Y, et al. A method integrating Taguchi, RSM and MOPSO to CNC machining parameters optimization for energy saving [J]. Journal of Cleaner Production, 2016, 135:263-275. 
[7]. Ghaffari-Nasab N, Amari S G, Ghazanfari M. A hybrid simulated annealing based heuristic for solving the location-routing problem with fuzzy demands [J]. Sciatica Ironical, 2013, 20(3):919-930.

[8]. Moon J Y, Shin K, Park J. Optimization of production scheduling with time-dependent and machine-dependent electricity cost for industrial energy efficiency [J]. International Journal of Advanced Manufacturing Technology, 2013, 68(1-4):523-535. 